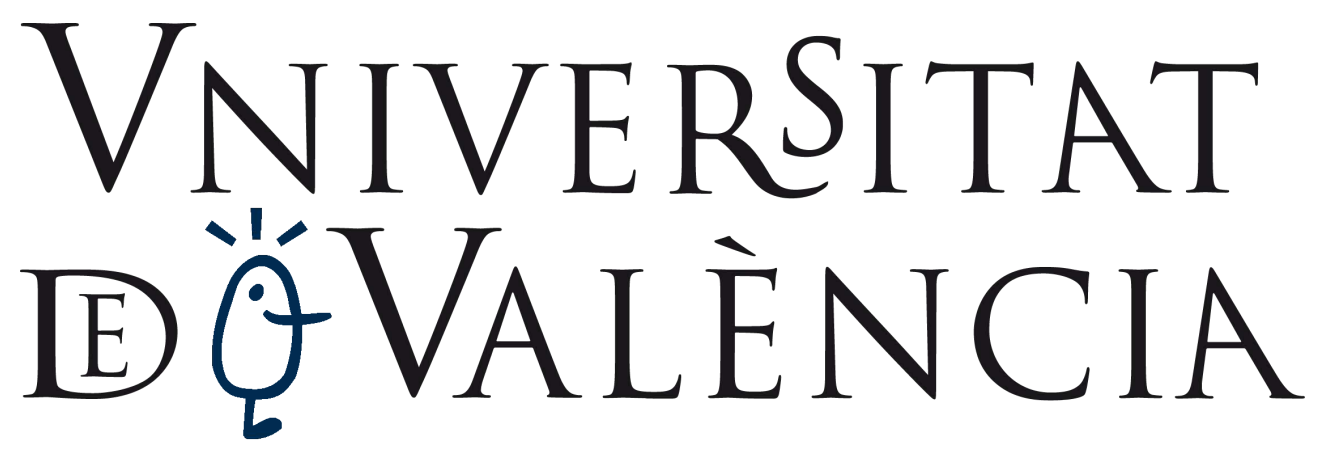

Citation for the original published paper:

Xin CHEN, Xiao-dong XU, Hong-jia LI, Xiao-feng TAO, Tommy Svensson, Carmen Botella, Improved resource allocation strategy in SU-CoMP network,

The Journal of China Universities of Posts and Telecommunications,

Volume 18, Issue 4, 2011, Pages 7-12, ISSN 1005-8885,

https://doi.org/10.1016/S1005-8885(10)60076-3.

The published paper can be obtained in the following link:

https://www.sciencedirect.com/science/article/pii/S1005888510600763

This is an author's version under creative common license CC-BY-NC-ND 4.0 


\title{
Improved resource allocation strategy in SU-CoMP network
}

\author{
CHEN Xin $^{1}(\bowtie)$, XU Xiao-dong ${ }^{1}$, LI Hong-jia ${ }^{1}$, TAO Xiao-feng ${ }^{1}$, Tommy Svensson ${ }^{2}$, Carmen Botella ${ }^{2}$ \\ 1. Wireless Technology Innovation Institute, Key Laboratory of Universal Wireless Communication, Ministry of Education, \\ Beijing University of Posts and Telecommunications, Beijing 100876, China \\ 2. Signal and Systems, Chalmers University of Technology, SE-412-96 Göteborg, Sweden
}

\begin{abstract}
Coordinated multi-point transmission and reception (CoMP) for single user, named as SU-CoMP, is considered as an efficient approach to mitigate inter-cell interference in orthogonal frequency division multiple access (OFDMA) systems. Two prevalent approaches in SU-CoMP are coordinated scheduling (CS) and joint processing (JP). Although JP in SU-CoMP has been proved to achieve a great link performance improvement for the cell-edge user, efficient resource allocation (RA) on the system level is quite needed. However, so far limited work has been done considering JP, and most existing schemes achieved the improvement of cell-edge performance at cost of the cell-average performance degradation compared to the single cell RA. In this paper, a two-phase strategy is proposed for SU-CoMP networks. CS and JP are combined to improve both cell-edge and cell-average performance. Compared to the single cell RA, simulation results demonstrate that, the proposed strategy leads to both higher cell-average and cell-edge throughput.
\end{abstract}

Keywords OFDMA system, SU-CoMP, RA, binary power allocation based multi-cell coordinated proportional fair scheduling (BP-CPF), sub-channels assignment for joint processing (JP-SA)

\section{Introduction}

As a key technique to reduce inter-cell interference (ICI) and further improve cell-edge performance for OFDMA systems, CoMP transmission and reception had been recently proposed in the 3rd generation partnership projects (3GPP) long term evolution (LTE) and LTE-advanced recently in Refs. [1-2].

In the downlink CoMP, two approaches are often considered, which are namely, CS and JP as in Ref. [3]. In the $\mathrm{CS}$, data to single user equipment (UE) is instantaneously transmitted from one cell and scheduling decisions are coordinated made among cells. In JP of SU-CoMP, data to a single UE is simultaneously transmitted from multiple cells.

In Refs. [4-8], CS has been studied without considering JP. Authors in Ref. [9] concluded that although SU-CoMP could benefit cell-edge performance, it cause a great cell-average throughput loss due to more system resource allocation to a particular user. In Refs. [10-12] frequency plan based

Corresponding author: CHEN Xin, E-mail: jiuchen1986@bupt.edu.cn schemes are proposed, in which the total system resources were pre-divided into non-CoMP and CoMP resource. In order to limit the loss of cell-average performance, JP is only allowed to use the CoMP resource. However, as the frequency plans were pre-defined, such RA schemes are lack of flexibility leading to a great loss of schedule gain.

In this paper, the RA problem is addressed under a SU-CoMP environment. The binary power allocation is used due to that the power is equally allocated between subcarriers in the downlink of LTE and LTE-advanced system. In Ref. [8], a distributed multi-cell binary power allocation scheme is proposed to maximize system capacity. Inspired by this work, a two-phase resource allocation strategy is proposed in this paper. In the first phase of proposed strategy, a binary power allocation based coordinated proportional fair scheduling (BP-CPF) without JP is adopted. Different from the scheme in Ref. [8] aiming at total system capacity maximization, the BP-CPF is used to maximize total system utility and also obtain the potential JP resources. According to the results of BP-CPF, a JP-SA is proposed in the second phase, which aims at further improving cell-edge throughput and minimizing the loss of cell-average. Simulation results 
show that proposed strategy provides improvement for both cell-average and cell-edge performance compared to PF scheduling with single cell transmission.

The rest of this paper is organized as follows. In Sect. 2, the system model for SU-CoMP network and the calculation of JP user throughput is given. Based on the model, a two-phase RA strategy for SU-CoMP is proposed in Sect. 3. The performance of proposed strategy is evaluated in Sect. 4. Then the Sect. 5 concludes the paper.

\section{System model}

\subsection{SU-CoMP network}

A downlink SU-CoMP network in a multi-cell OFDMA system is considered in this paper as be shown in Fig. 1. It is assumed that $N$ single-antenna cells and $K$ single-antenna users existing in the system. A frequency reuse factor of 1 is considered and $L$ sub-channels are available in each cell. The $K$ users are uniformly distributed in the system with index of $k=1,2, \ldots, K$. And $K_{n}$ denotes the set of users in cell $n=1,2, \ldots, N$. In this paper, two types of sets of cells are defined as follow:

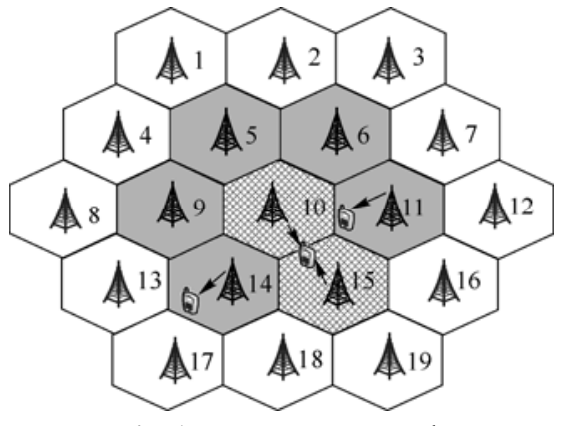

Fig. 1 SU-CoMP network

Coordinated cell set (CCS): Each cell in the same CCS shares the channel state information (CSI) and applies CS in the first phase of proposed strategy. Different CCSs can be either overlap or disjoint and no coordination among cells in disjoint CCSs. As illustrated in Fig. 1, cells 5, 6, 9-11, 14 and 15 could compose a CCS.

Joint processing set (JPS): Multiple cells belonging to the same JPS could use JP to serve the same user in the second phase of proposed strategy. In this paper, different JPSs are assumed to be disjoint. In Fig. 1, cell 10 ad 15 could compose a JPS.

Herein, we don't constrain the relationship between CCS and JPS. A JPS can be either included in a CCS (as shown in Fig. 1) or not. For simplicity, it is assumed that CCS and JPS are pre-defined by the network and fixed during the period of scheduling, i.e. the cell selection for CoMP is not considered here. And the number of cells in each JPS is limited to 2 . We define $\Phi$ and $\Omega$ as the cells included in the CCS and JPS respectively.

Note that different number of cells included in CCS and JPS may have a great impact on the RA strategy and CoMP performance as well as application issues. Although this is beyond the scope of this paper, we will give a performance comparison of different size of CCS in the simulation results.

\subsection{Throughput calculation}

A multi-path fading radio channel is assumed including pathloss, shadow fading and frequency selective fading. $G_{n, k}^{l}$ represents the channel gain from cell $n$ to user $k$ on sub-channel $l$. It is supposed that the coherence time of the channel is longer than the scheduling period and perfect CSI is available at the receiver and transmitter. In conventional single cell transmission, each user is served by only one cell at the same time. In this case the throughput of user $k$ serving by cell $n$ on sub-channel $l=1,2, \ldots, L$ at time $t$ using single cell transmission is given by

$$
R_{n, k}^{l}(t)=B \operatorname{lb}\left(1+\frac{G_{n, k}^{l} P_{n, l}(t)}{\sigma^{2}+\sum_{i \neq n}^{N} G_{i, k}^{l} P_{i, l}(t)}\right)
$$

where $\sigma^{2}$ is the power of the additive white Gaussian noise and $B$ is the sub-channel bandwidth, $P_{n, l}(t)$ denotes the power used by cell $n$ on sub-channel $l$ at time $t$. Assuming an equal power allocation between sub-channels and a binary power control, $P_{n, l}(t) \in\{0, P\}$, where $P$ is the peak power constraint of each sub-channel, $\sum_{i \neq n}^{N} G_{i, k}^{l} P_{i, l}(t)$ is the total co-channel interference from other cells. In JP, several cells can serve a single user using same time-frequency resource. Assuming a non-coherent reception at the user, the throughput of user $k$ with JP on sub-channel $l$ is given as

$$
R_{\Omega, k}^{l}(t)=B \mathrm{lb}\left(1+\frac{\sum_{n \in \Omega} G_{n, k}^{l} P_{n, l}(t)}{\sigma^{2}+\sum_{i \notin \Omega}^{N} G_{i, k}^{l} P_{i, l}(t)}\right)
$$

where $\sum_{n \in \theta_{n}} G_{n, k}^{l} P_{n, l}(t)$ is the sum of signal from JP cells in $\Omega$ with equal gain combining at the receiver. It should be pointed out that only spatial diversity is considered here, and the user throughput can be further improved if space 
multiplexing is applied.

\section{SU-CoMP resource allocation}

In this section, a low complexity and semi-distributed strategy is proposed to improve both cell-edge and cell-average performance. The proposed strategy consists of two phases:

1) Binary power allocation based multi-cell coordinated proportional fair scheduling (BP-CPF) without JP for system utility maximization.

2) Sub-channels assignment for JP (JP-SA). In the first phase, the BP-CPF is utilized to guarantee cell-average performance and obtain potential JP resource. In the second phase, on the basis of the BP-CPF, sub-channels for JP are selected and assigned to JP users aiming to improve cell-edge throughput. The details of each phase are described as follows.

\subsection{Phase 1: BP-CPF}

The objective utility function adopted here is user's long-term proportional fairness factor, i.e. $U_{k}\left(R_{k}(t)\right.$, $\left.\bar{R}_{k}(t)\right)=R_{k}(t) / \bar{R}_{k}(t)$ for user $k$, where $R_{k}(t)$ and $\bar{R}_{k}(t)$ denote instantaneous throughput and average throughput of user $k$ respectively. Defining $\boldsymbol{P}_{l}(t)=\left[P_{1, l}(t), P_{2, l}(t), \ldots\right.$, $\left.P_{n, l}(t), \ldots, P_{N, l}(t)\right]$ as the power vector on sub-channel $l$ at time $t, R_{n, k}^{l}(t)$ in Eq. (1) can be rewritten as $R_{n, k}^{l}\left(\boldsymbol{P}_{l}(t)\right)$. Therefore the $R_{k}(t)$ is given by

$$
R_{k}(t)=\sum_{l} R_{n, k}^{l}\left(\boldsymbol{P}_{l}(t)\right) ; \quad k \in K_{n}
$$

and the average throughput $\bar{R}_{k}(t)$ can be expressed using an exponentially low-pass time window as follow:

$\bar{R}_{k}(t)=\left(1-\rho_{w}\right) \bar{R}_{k}(t-1)+\rho_{w} R_{k}(t)$

where $\rho_{\mathrm{w}}=\left(T_{\mathrm{s}} / T_{\mathrm{w}}\right), T_{s}$ is the slot length, and $T_{\mathrm{w}}$ is the

length of the window. Thus the system utility maximization problem can be formulated as follows:

$$
\begin{aligned}
& \max \sum_{k=1}^{K} U_{k}\left(R_{k}(t), \bar{R}_{k}(t)\right) \\
& \text { s.t. } \quad P_{n, l}(t) \in\{0, P\}
\end{aligned}
$$

The Eq. (6) ensures the power allocation is binary. $P_{n, l}(t)=0$ denotes that cell $n$ is inactive on sub-channel $l$, otherwise sub-channel $l$ is active in the cell $\mathrm{n}$. It is also assumed that each sub-channel can be allocated to only one user.

Define $\quad U_{k}^{l}\left(\boldsymbol{P}_{l}(t)\right)=R_{n, k}^{l}\left(\boldsymbol{P}_{l}(t)\right) / \bar{R}_{k}(t) ; k \in K_{n} \quad$ as the utility obtained by user $k$ on sub-channel $l$. Therefore, in order to maximize system utility, a cell $n_{0}$ should be activated on sub-channel $l$ if this action results in an increase in utility, i.e.

$\sum_{n=1}^{N} U_{k_{n}^{l}(t)}^{l}\left(\boldsymbol{P}_{l}(t) \mid P_{n_{0}, l}(t)=P\right)>\sum_{n \neq n_{0}}^{N} U_{k_{n}^{l}(t)}^{l}\left(\boldsymbol{P}_{l}(t) \mid P_{n_{0}, l}(t)=0\right)$

where

$k_{n}^{l}(t)=\underset{k \in K_{n}}{\arg \max } U_{k}^{l}\left(\boldsymbol{P}_{l}(t) \mid P_{n, l}(t)=P\right)$

The left part of Eq. (7) is the sum of system utility obtained with cell $n_{0}$ activated on sub-channel $l$, while the right part represents the utility with cell $n_{0}$ deactivated. Eq. (8) indicates that the selected user $k_{n}^{l}(t)$ is the user with the best utility on sub-channel $l$ in cell $n$. And the corresponding utility of the user is $k_{n}^{l}(t)$ the exact largest utility the cell $n$ could get.

Eq. (7) requires information sharing across the whole network. It is known that coordination among entire network is impossible due to high computational burden and large signaling overhead. Note that interference from very far cells is negligible. Therefore, we limit the coordination only in the CCS of cell $n_{0}$ to reduce overhead with a little performance decreasing. Define $\Phi_{n_{0}}$ as the cells of CCS including cell $n_{0}$, the procedures of BP-CFP is given in Algorithm A1. An iteration approach is adopted. Starting with full power allocation, each cell remains active or inactive on each sub-channel at every iteration and updates the power allocation. The algorithm is run until the total system utility stabilize or for a given number of iterations. Steps of Algorithm A1 are elaborated as follows:

Step 1 Initialize $P_{n, l}^{(i=1)}(t)=P \forall n, l$, select $\Phi_{n}$ for each cell $n$.

Step 2 Each iteration $i$, for all cells and sub-channels.

Step 3 Select scheduling user $k_{n}^{l}(t)=\underset{k}{\arg \max } U_{k}^{l}$. $\left(\boldsymbol{P}_{l}^{(i)}(t) \mid P_{n, l}^{(i)}(t)=P\right)$.

Step 4 If $\sum_{i \in \Phi_{n}}^{N} U_{k_{i}^{l}}^{l}\left(\boldsymbol{P}_{l}^{(i)} \mid P_{n, l}^{(i)}=P\right)>\sum_{i \neq n, i \in \Phi_{n}}^{N} U_{k_{i}^{l}}^{l}\left(\boldsymbol{P}_{l}^{(i)} \mid P_{n, l}^{(i)}=0\right)$, then $P_{n, l}^{(i+1)}=P$, otherwise $P_{n, l}^{(i+1)}=0$.

Step 5 Repeat Steps 1-4 until the total system utility stabilize or for a given iteration time.

\subsection{Phase 2: JP-SA}

The multi-cell power allocation result obtained in BP-CPF forms a sub-channel reuse pattern among all cells on each sub-channel. Fig. 2 illustrates a possible pattern. In second phase with pre-defined JPSs, sub-channels are assigned for JP based on the sub-channel reuse pattern obtained in BP-CPF. 


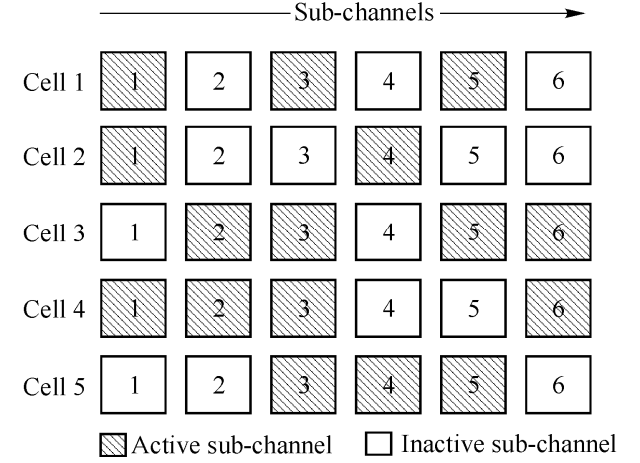

Fig. 2 A possible sub-channels reuse pattern after BP-CPF

Note that, in downlink OFDMA system with binary power allocation, only the active sub-channels are able to cause ICI. Hence, ICI only depends on the power status, and there will be no impact on other cells' performance when one or several cells change their scheduled users with their sub-channels power allocation fixed. According to the analysis, we propose that after the first phase, the sub-channels on which all the cells belonging to the same JPS are active can be considered as potential sub-channels for JP. For example, as illustrated in Fig. 2, assuming cell 3 and 4 construct a JPS, then sub-channel 2, 3 and 6 are JP potential sub-channels. Those potential sub-channels can be used for JP to improve cell-edge performance of cells in JPS without diminishing other cells' performance outside the JPS.

With the potential JP sub-channels, a JP-SA algorithm is proposed. First a candidate JP user is selected for each potential JP sub-channel. Assuming cell $n_{1}$ and cell $n_{2}$ compose a JPS $\Omega$, for the potential JP sub-channel $l$, a candidate JP user is selected from all the users in both cell $n_{1}$ and cell $n_{2}$ by

$$
k_{\Omega}^{l}=\underset{k \in K_{n_{1}} \cup K_{n_{2}}}{\arg \max } \frac{R_{\Omega, k}^{l}}{\bar{R}_{k}{ }^{\alpha}} ; \quad \alpha>1
$$

where $R_{\Omega, k}^{l}$ is calculated by Eq. (2) and $\alpha$ is a JP factor.

As mentioned before, the candidate JP users must be the exact cell-edge users which have poor long-term performance. From Eq. (9), it can be seen that as the $\alpha$ increases, the poorer average throughput the user has, the higher probability the user will be selected. And $\alpha>1$ guarantees that the cell-edge users even have more opportunities to be served than in phase 1 . The $\alpha$ can be dynamically adjusted according to system requirements. A cell-edge user is defined as the user with poor long-term performance.

Note that a throughput decrease is inevitable for some users scheduled in phase 1. To avoid excessive degradation of the system utility, the actual JP user must satisfy:
$\bar{R}_{k_{\Omega}^{\prime}} \leqslant \min \left\{\min _{n \in \Omega} \bar{R}_{k_{n}^{l}}, \bar{R}_{\mathrm{th}}\right\}$

where $k_{n}^{l}$ denotes the original user scheduled in phase 1 on sub-channel $l$ of cell $n . \bar{R}_{\mathrm{th}}$ is a pre-defined threshold used to ensure that the user really experience a poor performance and exactly needs JP. Meanwhile the actual JP user's long term performance must be no larger than all the original users scheduled in phase 1 to avoid excessive damage to the system utility. Therefore a user will be scheduled as an actual JP user only if inequality (10) holds. Otherwise the schedule results obtained in phase 1 will not be changed. Each JPS can select all its JP users and corresponding sub-channels to apply JP. Based on the description, a 2-step JP sub-channel assignment algorithm is obtained as follow:

Step 1 Each pre-defined JPS selects its candidate JP users on potential JP sub-channels according to Eq. (9).

Step 2 Each JPS selects actual JP users from candidate JP users according to Eq. (10) and assigns corresponding sub-channels to apply JP.

\section{Performance analysis}

Monte-Carlo simulations to measure performance of the proposed strategies are carried out. A cellular layout with 27 cell sites is considered with $500 \mathrm{~m}$ cell radius. The pathloss model is set as $L=128.1+37.6 \lg 10 \mathrm{~d}(\mathrm{~dB})$. Lognormal shadowing is considered with correlation distance $50 \mathrm{~m}$. SCME [13] is adopted for fast fading. Total system bandwidth is $10 \mathrm{MHz}$. Users are uniformly dropped in whole network.

All the CCS and JPS are assumed to be pre-defined. The size of each JPS is set to be 2 . It is assumed that users are uniformly dropped in whole network and equal power allocation among sub-channels. The threshold $\bar{R}_{\text {th }}$ in Eq. (10) is defined as the long-term average throughput of the worst $5 \%$ performance user in BP-CPF.

We compare our strategy with conventional single cell proportional fairness (PF) schedule. For single cell PF, based on just local information, each cell allocates sub-channels to users according to proportional fairness until all sub-channels have been allocated (full reuse). In addition, the comparison between the BP-CPF and JP-SA is given in the following Figures, along with different size of CCS.

Fig. 3 shows the comparisons of cumulative distribution function (CDF) of users' average throughput for single cell PF, BP-CPF and JP-SA under 40 users per cell. '3-cell CCS' means the number of cells included in each CCS is 3 . It can be observed that, BP-CPF and JP-SA both have a better 
performance than PF. The results will be demonstrated more explicitly in Figs. 4-5.

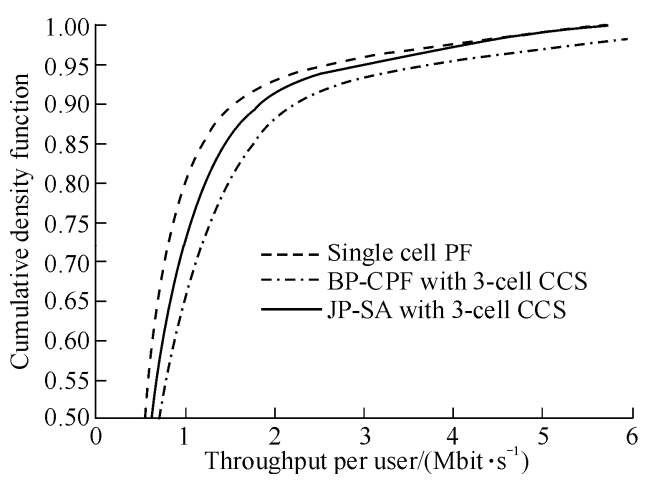

Fig. 3 The throughput CDF of users

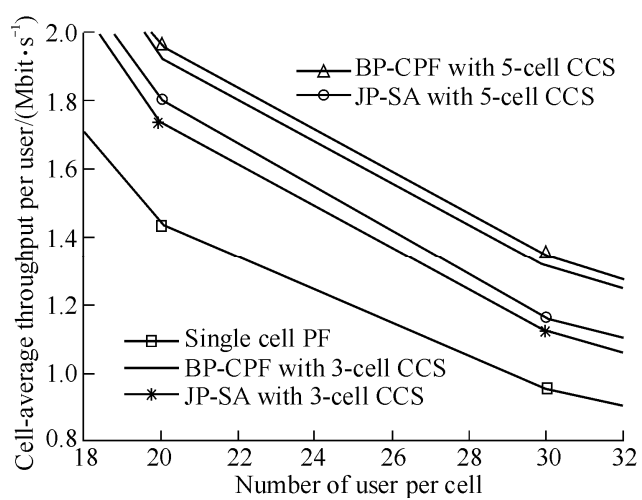

Fig. 4 Average throughput per user vs. number of user per cell

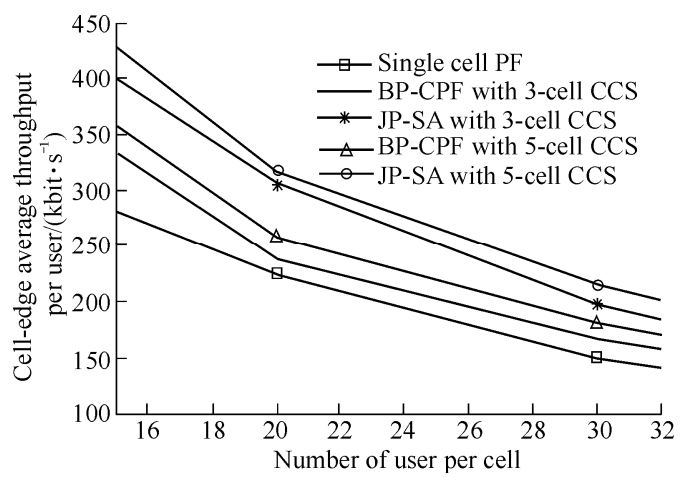

Fig. 5 Cell-edge average throughput per user vs. number of user per cell

In Fig. 4, the average throughput per user for the test algorithms versus number of users per cell is given, which reflects the cell-average performance of each algorithm. Similar to '3-cell CCS', '5-cell CCS' indicates that there are 5 cells in each CCS. It is evident that both BP-CPF and JP-SA achieve higher average user throughput than single cell PF. BP-CPF achieves the highest average throughput while JP-SA decreases a little, that probably because JP-SA 'grab' some sub-channels, which were allocated to cell-center users with better channel states in BP-CPF, to apply JP for cell-edge users with poor channel states. It also can be seen that larger size of CCS leads to further improvement for both BP-CPF and JP-SA due to more cells participate in coordination at the expense of complexity and overhead increase. Note that user average throughput decreases as the number of user increases for all test algorithms due to more users compete for resource.

Cell-edge throughput per user versus number of users per cell is illustrated in Fig. 5. It is calculated from the average throughputs among $5 \%$ most poor performance users. It can be seen that a better cell-edge throughput can be achieved by proposed strategy compared to single cell $\mathrm{PF}$ due to considering ICI mitigation in power allocation. We can also see that, with providing JP for cell-edge users based on BP-CPF, JP-SA further reduces the ICI and improves channel qualities for cell-edge users resulting in best cell-edge performance at the cost of cell-average throughput decrease as showed in Fig. 3. Note that more cells being involved in coordination also gives further improvement for cell-edge performance. Similar to Fig. 3, performance degrades as the number of user increases for all test algorithms.

Fig. 6 describes the comparison of ratio of power consumption for single cell $\mathrm{PF}, \mathrm{BP}-\mathrm{CPF}$ in 3-cell CCS and BP-CPF in 5-cell CCS (BP-CPF and JP-SA have same power consumption) under different cell load conditions. Combined with Figs. 3-5, it can be concluded that, compared to full reuse case, proposed strategy in this paper has a better performance with lower power consumption. This demonstrates the efficiency and necessity of coordinated power allocation. Note that larger size of CCS provides higher power efficiency. Besides, the power consumption increases as the users' number increases.

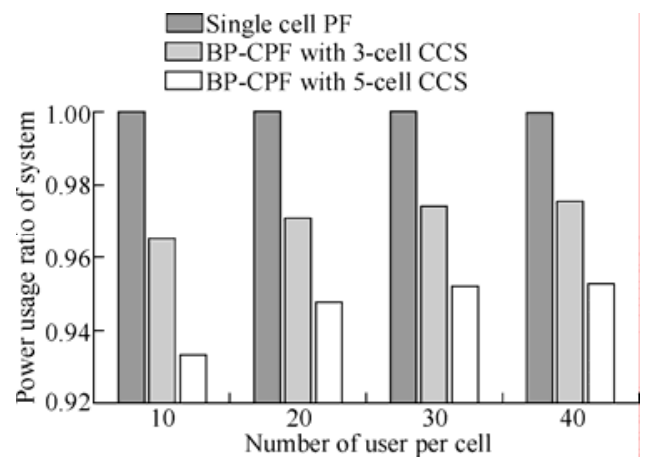

Fig. 6 Power usage ratio of network vs. number of user per cell

\section{Conclusions}

In this paper, resource allocation SU-CoMP network is considered and a two-phase RA strategy combined CS and JP 
is proposed. In phase 1, a binary power allocation based coordinated proportional fair scheduling (BP-CPF) is adopted to maximize system utility and obtain potential JP resources. Then, a sub-channel assignment scheme for JP (SA-JP) is proposed in phase 2 to further improve cell-edge performance. In comparison with conventional algorithm, better performance for both cell-edge and cell-average is obtained from the proposed strategy. Since we simply apply space diversity gain and static constructing to scale joint transmission, other joint transmission schemes will be studied on system level in future as well as dynamical constructing. In addition, simpler multi-cell binary power allocation for phase 1 is needed to reduce complexity and overhead of entire strategy.

\section{Acknowledgements}

This work was supported by the National Natural Science Foundation of China (61001116), State Emphasis Special Project 2009ZX03003-011-02, the Hi-Tech Research and Development Program of China (2009AA011506), and International Scientific and Technological Cooperation Program (2010DFA11060).

\section{References}

1. 3GPP TR 25.814. Physical layer aspects for evolved UTRA (Release 7), v7.1.0. 2006

2. 3GPP TR 36.814. Further advancements for E-UTRA physical layer aspects (Release 9), v9.0.0. 2010

3. Ericsson. A discussion on some technology components for LTE-advanced. 3GPP TSG RAN WG1 Meeting \#53. May 14, 2008,
Kansas City, MO, USA. 2008: R1-082024

4. Li G Q, Liu H. Downlink radio resource allocation for multi-cell OFDMA system. IEEE Transactions on Wireless Communications, 2006, 5(12): 3451-3459

5. Saraydar C U, Mandayam N B, Goodman D J. Pricing and power control in a multi-cell wireless data networks. IEEE Journal on Selected Areas in Communications, 2001, 19(10): 1883-1892

6. Zhu H, Zhu J, Liu K J R. Non-cooperative resource competition game by virtual referee in multi-cell OFDMA networks. IEEE Journal on Selected Areas in Communications, 2007, 25(6): 1079-1090

7. Chen L, Chen W W, Zhang X, et al. Inter-cell coordinated resource allocation for mobile WiMAX system. Proceedings of the IEEE Wireless Communications and Networking Conference (WCNC'09), Apr 5-8, 2009, Budapest, Hungary. New York, NY, USA: IEEE, 2009: 6p

8. Kiani S G, Øien G E, Gesbert D. Maximizing multicell capacity using distributed power allocation and scheduling. Proceedings of the IEEE Wireless Communications and Networking Conference (WCNC'07), Mar 11-15, 2007, Hong Kong, China. Piscataway, NJ, USA: IEEE, 2007: 1690-1694

9. Samsung. Further discussion on inter-cell inerference mitigation throughput limited coordination. 3GPP TSG RAN WG1 Meeting \#54bis, Sep 29-Oct 3, 2008, Prague, Czech. 2008: R1-083569

10. Samsung. Discussion on CoMP-SU-MIMO. 3GPP TSG RAN WG1 Meeting \#56, Feb 9-13, 2009, Athens, Greece. 2009: R1-090613

11. Potevio. Further discussion of frequency plan scheme on CoMP-SU-MIMO. 3GPP TSG RAN WG1 Meeting \#56bis, Mar 23-27, 2009, Seoul, Republic of Korea. 2009: R1-091415

12. Li J Y, Zhang H, Xu X D, et al. A novel frequency reuse scheme for coordinated multi-point transmission. Proceedings of the 71st Vehicular Technology Conference (VTC-Spring'10), May 16-19, 2010, Taipei, China. Piscataway, NJ, USA: IEEE, 2010: 5p

13. Baum D S, Hansen J, Salo J. An interim channel model for beyong-3G systems: Extending the 3GPP spatial channel model (SCM). Proceedings of the 61st Vehicular Technology Conference (VTC-Spring'05): Vol 5, May 30-Jun 1, 2005, Stockholm, Sweden. Piscataway, NJ, USA: IEEE, 2005: 3132-3136 